\title{
Predictive Cruise Control in Hybrid Electric Vehicles
}

\author{
Thijs van Keulen ${ }^{1}$, Gerrit Naus ${ }^{1}$, Bram de Jager ${ }^{1}$, \\ René van de Molengraft ${ }^{1}$, Maarten Steinbuch ${ }^{1}$, Edo Aneke $^{2}$ \\ ${ }^{1}$ Eindhoven University of Technology, P.O. Box 513, 5600 MB, Eindhoven, \\ The Netherlands (Tel: +31 40247 4828; e-mail: t.a.c.v.keulen@tue.nl). \\ ${ }^{2}$ TNO business unit Automotive, P.O. Box 756, 5700 AT, Helmond
}

\begin{abstract}
Deceleration rates have considerable influence on the fuel economy of hybrid electric vehicles. Given the vehicle characteristics and actual/measured operating conditions, as well as upcoming route information, optimal velocity trajectories can be constructed that maximize energy recovery. To support the driver in tracking of the energy optimal velocity trajectory, automatic cruise control is an important driver aid. In practice, perfect tracking of the optimal velocity trajectory is often not possible. An Adaptive Cruise Control (ACC) system is employed to react to the actual traffic situation. The combination of optimal velocity trajectory construction and ACC is presented as Predictive Cruise Control (PCC).
\end{abstract}

Keywords: HEV (Hybrid Electric Vehicle), regenerative braking, truck, energy consumption, optimization

\section{Introduction}

Hybrid Electric Vehicles (HEVs) save fuel by reusing kinetic and potential energy, that is recovered and stored during braking or driving down hill. The amount of energy recovered depends heavily on the followed deceleration trajectories. Taking HEV characteristics and current vehicle operating conditions into account, velocity trajectories can be determined that maximize the energy recovery [9]. This is especially beneficial for heavy-duty HEV because of the large variability of vehicle mass; a truck can be loaded or unloaded changing its mass by a factor 2-3.

The route information consists of velocity limitations, road curvature and road grade. This information can be derived from Geographical Information Systems (GIS) in combination with a routeplanner, and a Global Navigation Satellitebased System (GNSS) with map-matching algorithms to locate the vehicle in the map [7, 12]. The relevant vehicle characteristics and operating conditions include the electric machine size, battery capacity, gear selection strategy, and vehicle mass. The vehicle mass can be estimated online using a parameter estimator $[11,18]$, the other parameters are available in the vehicle. Considering a driver, he does not exactly know the optimal trajectory, therefore, tracking the optimal velocity trajectories will be difficult. Besides, the trajectories can vary considerably, due to changes in operating conditions and expected route characteristics. Furthermore, perfect tracking of an optimal velocity trajectory will not be possible in actual traffic. Therefore, an Adaptive Cruise Control (ACC) system may be employed, comprising automated Cruise Control (CC) as well as automated following of preceding traffic [14]. The automated CC facilitates tracking of the optimal velocity trajectories. Furthermore, the ACC will automatically adjust the vehicle velocity to actual preceding traffic.

In literature, several useful contributions can be found regarding velocity trajectory optimization in conventional vehicles. In [13] it is suggested to use dynamic programming to numerically solve the optimal velocity trajectory problem in hilly environment. This approach is successfully adapted in [4] using Model Predictive Control (MPC) in combination with an automated CC. In [17] Pontryagin's Minimum Principle is used to obtain an optimal velocity trajectory. However, these contributions have a high computational load, or/and do not discuss HEV characteristics nor the influence of traffic disturbance. 
The contribution of this paper consists of; i) presenting analytical solutions for the velocity trajectory optimization problem in HEVs; ii) combining the determination of optimal velocity trajectories for HEV and an ACC system. This enables automatic following of these trajectories as well as anticipation of disturbances by actual traffic. The combination is presented as Predictive Cruise Control (PCC). Besides, it is suggested to use part of the ACC system to assist the driver by indicating the optimal control action, at moments that full ACC is practically impossible. For instance by applying force feedback on the brake pedal [1], or visual indication on the dashboard.

The remainder of this paper is organized as follows; Section 2 presents a model of heavy-duty HEV longitudinal dynamics and drive train components; Section 3 discusses the construction of an optimal velocity trajectory; Section 4 details the ACC system; Section 5 integrates the velocity trajectory construction and the ACC, in the PCC setup; Section 6 shows simulation results; finally, in Section 7 and 8 we conclude and look forward.

\section{Vehicle model}

This paper uses a medium sized heavy-duty parallel HEV as carrier. The topology of the drive train components in a parallel hybrid configuration is shown in Fig. 1. The vehicle model takes into account the longitudinal dynamics. Static nonlinear maps describe the efficiency of combustion engine, electric machine and battery. The engine and electric machine are situated in front of a six speed automated gearbox and run with the same rotational velocity.

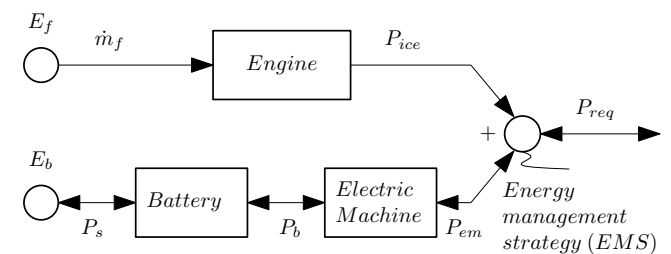

Figure 1: Hybrid drive train topology. Legend: $E_{f}$ is the stored fuel, $\dot{m}_{f}$ is the fuel mass flow, $P_{i c e}$ is the engine mechanical power, $E_{b}$ is the stored energy in the battery, $P_{s}$ is the effective storage power, $P_{b}$ is the electric power, $P_{e m}$ is the electric machine mechanical power, $P_{r e q}$ is the power request.

\subsection{Vehicle dynamics}

A basic model of vehicle longitudinal dynamics, including rolling resistance, gearbox and differential drag force, gravitational force and aerodynamic drag, can be found in $[3, \mathrm{p} .14]$, or in $[5$, p. 23].
The vehicle's road load force is described by;

$$
\begin{array}{r}
F_{r l}=c_{r} m g \cos \beta(x)+c_{d} v(t)+c_{d 0} \\
+c_{g b} v(t)+c_{g b 0}+\frac{1}{2} \rho_{a} A_{f}\left(c_{a}+k\right) v_{y}^{2} \\
+\frac{1}{2} \rho_{a} A_{f} c_{a}\left(v(t)+v_{x}(x)\right)\left|v(t)+v_{x}(x)\right|
\end{array}
$$

Here, $c_{r}$ is the rolling resistance coefficient, $m$ is the vehicle mass, $g$ is the gravitational constant $\beta(x)$ is the road grade as function of traveled distance $x, c_{d}$ is the differential loss coefficient, $v$ is velocity, $c_{d 0}$ is the differential loss force, $c_{g b}$ is the gearbox loss coefficient, $c_{g b 0}$ is the gearbox loss force, $\rho_{a}$ is the air density, $A_{f}$ the vehicle frontal area, $c_{a}$ the aerodynamic coefficient, $v_{x}$ is the wind velocity perpendicular to the vehicle forward velocity as function of traveled distance, $v_{y}$ is the wind velocity tangential to the vehicle forward velocity as function of traveled distance, and $k$ is the crosswind drag coefficient. The model only holds for vehicle forward velocities. Besides a road load force, the vehicle experiences a gravitational force;

$$
F_{g}=m g \sin \beta(x)
$$

The longitudinal vehicle dynamics are described by Newton's second law of motion using a force balance at the vehicle wheels;

$$
\begin{aligned}
m_{e} \frac{d v(t)}{d t} & =\frac{\left[T_{i c e}+T_{e m}+T_{s e r}\right] i_{g b} i_{f}}{r_{e}} \\
& -F_{g}-F_{r l}
\end{aligned}
$$

In which $m_{e}$ is the effective vehicle inertia including the rotational inertia of the drive train (a constant value for $m_{e}$ is used, hereby disregarding inertia fluctuations due to gear setting), $T_{i c e}$ is the resultant drive/brake torque of the engine and exhaust brake, $T_{e m}$ is the drive/brake torque of the electric machine, $T_{s e r}$ is the resulting brake torque of the service brakes, $r_{e}$ is the effective tire radius, $i_{g b}$ is the current gear ratio depending on vehicle velocity and power request, $i_{f}$ is the final drive ratio.

\subsection{Diesel engine}

The prime mover of the vehicle is a diesel engine, with a maximum power of $136 \mathrm{~kW}$. The engine is modeled as a power converter, relating the engine output power $P_{i c e}$ to fuel rate $\dot{m}_{f}$, see Fig. 2 . The different lines show the influence of engine rotational velocity. Besides, the engine is bounded by torque as function of rotational velocity, see Fig. 4.

\subsection{Electric machine}

The HEV has an electric machine as secondary power converter, with a maximum power of 44 $\mathrm{kW}$. The electric machine is also modeled as a power converter, relating the electric power $P_{b}$ 


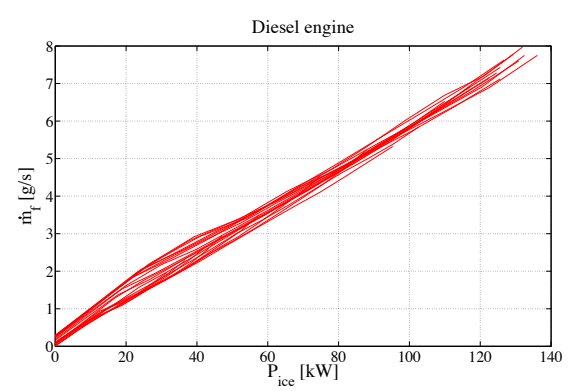

Figure 2: Diesel engine, fuel $\dot{m}_{f}$ to mechanical $P_{i c e}$ power conversion for different rotational velocities.

and mechanical power $P_{e m}$, see Fig. 3. The electric machine can work both as a motor and as a generator. At low rotational velocities the electric machine is limited by maximum torque, while at higher velocities the electric machine is limited by maximum power, see Fig. 4.

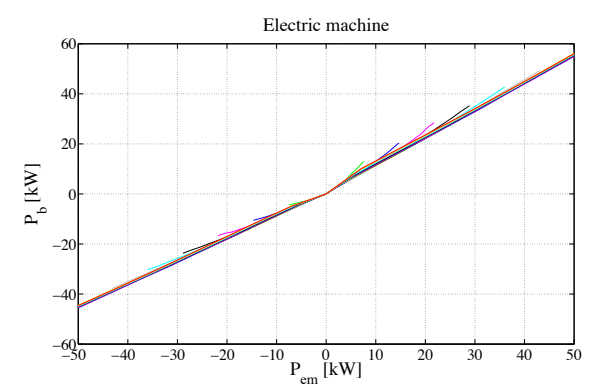

Figure 3: Electric machine, electric $P_{b}$ to mechanical $P_{e m}$ power conversion for different rotational velocities.

\subsection{Battery}

The lithium-ion battery used in the model has a maximum capacity of $9 \mathrm{MJ}$. The battery has losses during charging and discharging. The battery is described with a power based model, see Fig. 5. Here $P_{s}$ is the power that is effectively stored/retrieved from the battery, $P_{b}$ is the electrical power going in/out the electric machine. The losses during charging differ from the losses during discharging. Thermal and transient effects are not considered, nor the influence of state-ofcharge.

\subsection{Energy management strategy}

The Energy Management Strategy (EMS) governs the re-use of the stored energy. Objective of the EMS is to optimally split the power request over the engine and electric machine, and prevent the battery from over or under charging. In

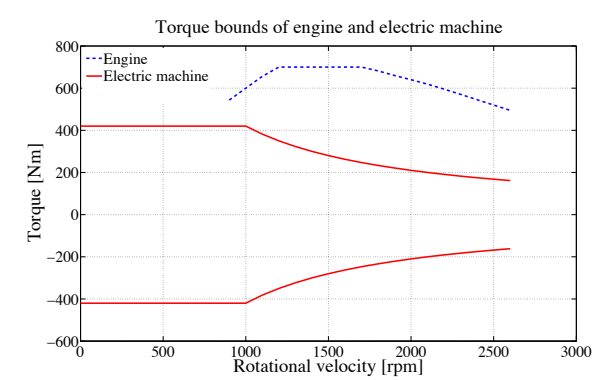

Figure 4: Torque bounds of engine and electric machine as function of rotational velocity.

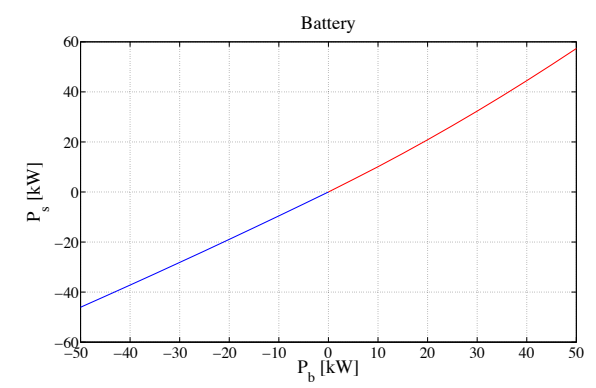

Figure 5: Battery, stored/retrieved power $P_{s}$ to electric $P_{b}$ power conversion.

this paper the focus is on construction and implementation of a velocity trajectory that maximizes the energy recovery, therefore the EMS is not discussed further here.

A general overview on EMS can be found in $[15,16]$. Several studies $[6,8,10]$, indicate a (small) performance increase by using a prediction of the future power trajectory. Using the PCC setup, a prediction of the future power trajectory is available, which could help in EMS optimization.

\section{Trajectory builder}

\subsection{Information systems}

Information systems, such as GIS in combination with GNSS, like Global Positions System (GPS), are available in modern vehicles. Mapmatching processes to locate the vehicle on the map together with a routeplanner provide information about the velocity limits, curvature and road grade of the upcoming route. This accommodates prediction of maximum allowable velocities, as well as stopping points, as a function of the traveled distance on the route. Furthermore, using the additional information provided by the radar and/or vision sensor, the relative position $x_{r}$, and velocity $v_{r}$, with respect to a preceding vehicle, might be used to adjust the route 
velocity trajectory to the actual traffic situation In this study it is assumed the vehicle mass is exactly known. The integration of a mass estimator in the simulation model is part of the future work.

\subsection{Route velocity trajectory}

Based upon GIS and traffic information it is possible to divide a route into $i$ segments with constant velocity limit and constant road grade as function of the traveled distance, see Fig. 6. Segments can also be divided by stopping points. The velocity limit is defined as the minimum of the maximum (legal) velocity, and the maximum cornering velocity. This approach is applied in the Route velocity trajectory block, see Fig. 11. Output of this block is a matrix of the form;

$v_{\text {set }}=\left[\begin{array}{ccccc}s_{\text {tot_1 }} & v_{0 \_1} & v_{\text {lim_ } 1} & v_{3 \_1} & \beta_{1} \\ s_{\text {tot_2 }} & v_{0 \_} 2 & v_{\text {lim_ } 2} & v_{3 \_} 2 & \beta_{2} \\ \vdots & \vdots & \vdots & \vdots & \vdots \\ s_{\text {tot } i} & v_{0 \_i} & v_{\text {lim } \_} & v_{3 \_i} & \beta_{i}\end{array}\right]$

Where $s_{\text {tot }} i$ is the segment length, $v_{0 \_i}$ is the segment initial velocity, $v_{3} i$ is the segment end velocity, $\beta_{i}$ is the segment road grade, $v_{\text {lim } i}$ is the velocity limitation in the segment, and $i$ indicates the number of segments. For the initialization the initial velocity of the first segment is corrected for the current vehicle velocity;

$$
v_{0 \_1}=\max \left(v_{0 \_1}, v_{c u r}\right)
$$

The matrix $v_{\text {set }}$ is input to the next block; the Optimal velocity trajectory block.

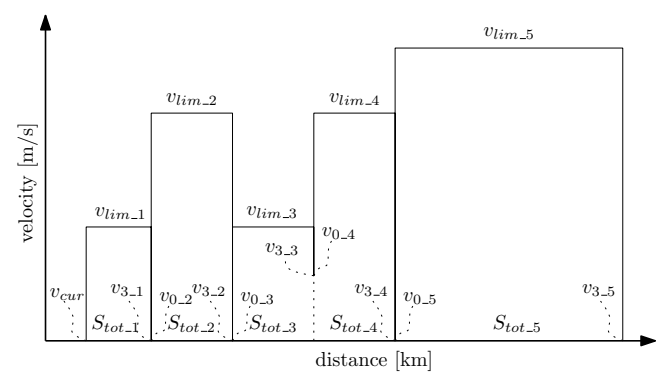

Figure 6: Route division into segments. In which $v_{c u r}$ is the current vehicle velocity, $v_{l i m_{-} i}$ is the velocity limit, $v_{0 \_i}$ the starting velocity, $v_{3 \_i}$ the end velocity, and $S_{\text {tot } i}$ the distance, of segment $i$.

\subsection{Optimal velocity trajectory}

Focusing on one segment, the velocity trajectory can be divided into an acceleration part, a constant velocity part and a deceleration part, see Fig. 7. In this section the calculation of the optimal velocity trajectory for such a vehicle is discussed. To enable calculation of the optimal velocity trajectory $\hat{v}_{\text {opt }}(x)$, a vehicle model and an estimation of the road-load force $\hat{F}_{r l}(v)$ are required. The road load force depends on the vehicle mass and the route characteristics. It is assumed that the vehicle conditions are known such that (1) and (2) are sufficiently accurately estimated by;

$$
\hat{F}_{r l}=c_{0}+c_{1} v+c_{2} v^{2}
$$

Here, $c_{0}$ is the drag force independent of vehicle velocity $v, c_{1}$ is the coefficient for drag force linearly depending on velocity, and $c_{2}$ is the coefficient for the drag force depending on velocity squared.

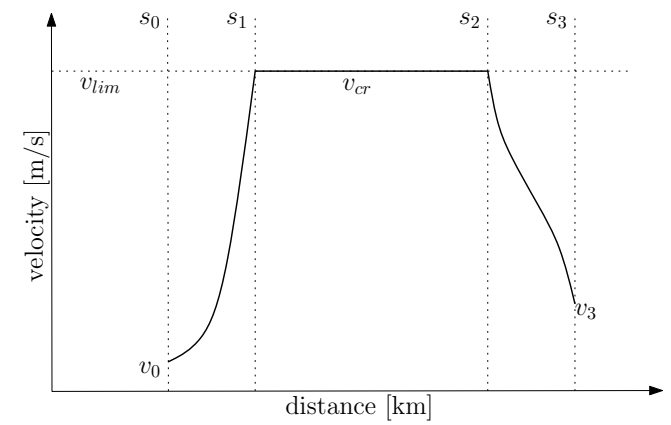

Figure 7: Route segment. In which $v_{0}$ is the initial velocity, $v_{l i m}$ is the velocity limit, $v_{3}$ is the end velocity, $s_{1}-s_{1}$ is the distance traveled in the acceleration part, $s_{2}-s_{1}$ is the distance traveled in the constant velocity part, and $s_{3}-s_{2}$ is the distance traveled in the deceleration part.

\subsection{1 acceleration path}

In [17] it is shown that full throttle accelerations lead to fuel optimal velocity trajectories. Fortunately, real-life driving behavior of heavy-duty vehicles is to use the full power of the vehicle, even for an unloaded vehicle, as was indicated in [2]. Therefore, this approach is especially relevant for heavy-duty vehicles. Assuming that the vehicle accelerates with constant motor power, hereby ignoring power discontinuities during gearshifts, the nett force at the vehicle wheels is described with;

$$
F_{m}=\frac{P_{\max }}{v}-c_{0}-c_{1} v-c_{2} v^{2}
$$

Here, $P_{\max }$ is the maximum available motor power during the acceleration phase. We assume that the measured relation between the produced engine power and the fuel consumption, see Fig. 2 , can be approximated with an affine relation;

$$
\dot{m}_{f} \approx \dot{m}_{f 0}+\frac{k_{i c e}}{h_{f}} P_{i c e}
$$


Here $\dot{m}_{f}$ is the approximated fuel mass flow, $\dot{m}_{f 0}$ is the fuel mass flow at zero torque, $k_{i c e}$ corresponds to the combustion efficiency and engine internal losses, and $h_{f}$ is the lower heating value of the fuel. The vehicle longitudinal equation of motion becomes;

$$
\frac{d v}{d t}=\frac{F_{m}}{\hat{m}_{e}}
$$

Here, $\hat{m}_{e}$ is the estimated effective vehicle mass, including the rotational inertia of all rotating parts. Rewriting (9), substituting (7), and integrating from the starting velocity $v_{0}$ to cruising velocity $v_{l i m}$ gives the acceleration time;

$$
\Delta t_{t_{0}}^{t_{1}}=m_{e} \int_{v_{0}}^{v_{l i m}} \frac{1}{F_{m}} d v
$$

This has the solution;

$$
\begin{aligned}
\Delta t_{t_{0}}^{t_{1}} & =m_{e} \sum_{n=1}^{3} \frac{R_{n} \ln \left(v_{l i m}-R_{n}\right)}{c_{0}+2 c_{1} R_{n}+3 c_{2} R_{n}^{2}} \\
& -m_{e} \sum_{n=1}^{3} \frac{R_{n} \ln \left(v_{0}-R_{n}\right)}{c_{0}+2 c_{1} R_{n}+3 c_{2} R_{n}^{2}}
\end{aligned}
$$

Here $R_{n}$ is the $n$th root of the cubic equation;

$$
-P_{\max }+c_{0} z+c_{1} z^{2}+c_{2} z^{3}=0
$$

This equation can be solved analytically. The fuel consumption, required during the acceleration part, is expressed algebraically in the start velocity $v_{0}$ and end velocity $v_{l i m}$

$$
m_{f}=\left.\left(\dot{m}_{f 0}+\frac{k_{i c e}}{h_{f}} P_{\max }\right) \Delta t\right|_{t_{0}} ^{t_{1}}
$$

The covered distance is calculated similarly by multiplying (11) on both sides with $v$. Solving this equation yields:

$$
\left.\Delta s\right|_{s_{0}} ^{s_{1}}=\left.R_{n} \Delta t\right|_{t_{0}} ^{t_{1}}
$$

Note that both $\left.\Delta t\right|_{t_{0}} ^{t_{1}}$ and $\left.\Delta s\right|_{s_{0}} ^{s_{1}}$ are functions of the form $x \ln y$. When the end velocity of the previous section is equal to the $v_{\text {lim }}$ of the current section, the acceleration part is ignored, and $\left.\Delta t\right|_{t_{0}} ^{t_{1}}$ and $\left.\Delta s\right|_{s_{0}} ^{s_{1}}$ are set to zero.

\subsection{2 deceleration path}

In [17] it is suggested that strong deceleration braking leads to fuel optimal velocity trajectories. However, this assumption is not valid for $\mathrm{HEV}$, since they can recover energy during the braking. The following equations for optimal deceleration path description are suggested. The electric machine has a constant torque bound $T_{e m}$ at low velocities $v<v_{r m}$ and a constant power bound $P_{e m}$ at high rotational velocities The nett force that decelerates the vehicle can then be expressed as:

$$
\begin{aligned}
F_{b r T}= & -\frac{\left(T_{\text {ser }}+\left(T_{e m}+T_{d r a g} i_{g b} i_{f}\right)\right)}{r_{w}} \\
& -c_{0}-c_{1} v-c_{2} v^{2}
\end{aligned}
$$

for $v<v_{r m}$, and

$$
\begin{aligned}
F_{b r P}= & -\frac{P_{e m}}{v}-\frac{\left(T_{s e r}+T_{d r a g} i_{g b} i_{f}\right)}{r_{w}} \\
& -c_{0}-c_{1} v-c_{2} v^{2}
\end{aligned}
$$

for $v \geqslant v_{r m}$. Here, $T_{e m}$ is the electric machine brake torque, $P_{e m}$ is the electric machine brake power, $T_{s e r}$ is the brake torque of the service brakes, $T_{d r a g}$ is the engine drag torque, $r_{w}$ is the wheel radius, $i_{g b}$ is the current gearbox ratio, $i_{f}$ is the final drive ratio. It is assumed that the vehicle does not change gear during the deceleration. Note that braking with $T_{e m}<T_{\text {em_max }}$, and $P_{e m}<P_{e m \_m a x}$, is in any case suboptimal, when disregarding the electric machine internal efficiency, and assuming that the battery is not fully charged. The value of $T_{e m}$ and $P_{e m}$ is known a priori. The deceleration time becomes;

$$
\begin{aligned}
\left.\Delta t\right|_{t_{2}} ^{t_{3}}= & m_{e} \int_{v_{l i m}}^{v_{r m}} \frac{1}{F_{b r T}} d v \\
& +m_{e} \int_{v_{r m}}^{v_{3}} \frac{v}{F_{b r P}} d v
\end{aligned}
$$

This has the solutions;

$$
\begin{aligned}
\left.\Delta t\right|_{t_{2}} ^{t_{r m}}= & m_{e} \sum_{m=1}^{2} \frac{R_{m} \ln \left(v_{r m}-R_{m}\right)}{c_{0}+2 c_{1} R_{m}+3 c_{2} R_{m}^{2}} \\
& -m_{e} \sum_{m=1}^{2} \frac{R_{m} \ln \left(v_{l i m}-R_{m}\right)}{c_{0}+2 c_{1} R_{m}+3 c_{2} R_{m}^{2}} \\
\left.\Delta t\right|_{t_{r m}} ^{t_{3}} & =m_{e} \sum_{n=1}^{3} \frac{R_{n} \ln \left(v_{3}-R_{n}\right)}{c_{0}+2 c_{1} R_{n}+3 c_{2} R_{n}^{2}} \\
& -m_{e} \sum_{n=1}^{3} \frac{R_{n} \ln \left(v_{r m}-R_{n}\right)}{c_{0}+2 c_{1} R_{n}+3 c_{2} R_{n}^{2}}
\end{aligned}
$$

Here $R_{m}$ is the $m$ th root of the quadratic equation;

$$
\begin{array}{r}
\left(\frac{T_{s e r}+\left(T_{e m}+T_{d r a g}\right) i_{g b} i_{f}}{r_{w}}+c_{0}\right) \\
+c_{1} z+c_{2} z^{2}=0
\end{array}
$$

and, $R_{n}$ is the $n$th root of the cubic equation;

$$
\begin{array}{r}
P_{e m}+\left(\frac{T_{s e r}+T_{d r a g} i_{g b} i_{f}}{r_{w}}+c_{0}\right) z \\
+c_{1} z^{2}+c_{2} z^{3}=0
\end{array}
$$


The covered distance is calculated similarly by multiplying (17) on both sides with $v$. Solving this equation gives;

$$
\begin{aligned}
\left.\Delta s\right|_{s_{2}} ^{s_{r m}} & =\left.R_{m} \Delta t\right|_{t_{2}} ^{t_{r m}} \\
\left.\Delta s\right|_{s_{r m}} ^{s_{3}} & =\left.R_{n} \Delta t\right|_{t_{r m}} ^{t_{3}}
\end{aligned}
$$

The recovered energy is;

$$
\begin{aligned}
E_{3} \approx & -\left.\eta_{e m} P_{e m} \Delta t\right|_{t_{2}} ^{t_{r m}} \\
& -\left.\eta_{e m} T_{e m} \Delta t\right|_{t_{r m}} ^{t_{3}}
\end{aligned}
$$

In which $\eta_{e m}$ is the linear approximation of the electric machine efficiency, as depicted in Fig. 3. The equivalent fuel consumption is;

$$
m_{f 3}=\frac{E_{3}}{h_{f}}
$$

Here it is assumed that setting $T_{\text {set }}=0$ leads to fuel optimal results. However, this is with the cost of larger traveling time. Future work will focus in solving the equations described above subject to a time constraint, possibly resulting in $T_{\text {ser }}>0$ and $v_{c r}<v_{l i m}$, with $v_{c r}$ the velocity on the constant velocity path.

\subsection{3 constant velocity path}

From the covered distance in the acceleration and deceleration path, follows the distance to be covered with constant velocity;

$$
\left.\Delta s\right|_{s_{1}} ^{s_{2}}=\left(s_{0}-s_{3}\right)-\left.\Delta s\right|_{s_{0}} ^{s_{1}}-\left.\Delta s\right|_{s_{2}} ^{s_{3}}
$$

The travel time in this part follows from

$$
\left.\Delta t\right|_{t_{1}} ^{t_{2}}=\frac{\left.\Delta s\right|_{s_{1}} ^{s_{2}}}{v_{l i m}}
$$

The power required to overcome the road load forces is;

$$
P_{\text {req }}=c_{0} v_{l i m}+c_{1} v_{\text {lim }}^{2}+c_{2} v_{\text {lim }}^{3}
$$

Using (8), the fuel consumption on the constant velocity path becomes;

$$
m_{f 2}\left(v_{l i m}\right)=\left.\left(\dot{m}_{f 0}+\frac{k_{i c e} P_{r e q}}{h_{f}}\right) \Delta t\right|_{t_{1}} ^{t_{2}}
$$

\subsection{4 trajectory construction}

The results obtained in the previous sections can be used to construct a velocity trajectory $\hat{v}_{o p t}(x)$, see Fig. 8. $\hat{v}_{\text {opt }}$ is obtained by constructing an equidistant grid $x$ with length equal to the total route distance, and numerically solving the inverse of (14), (22), (23) and (26). Furthermore, a required power trajectory $\hat{P}_{r e q}(t)$ and fuel consumption $m_{f}$ can be estimated for the upcoming route. The required power trajectory can be used by the Energy Management Strategy (EMS), as already discussed in Section 2.5.

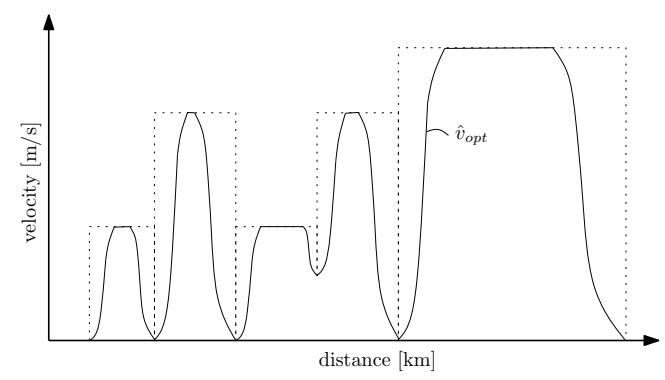

Figure 8: Calculated optimal velocity trajectory $\hat{v}_{\text {opt }}$.

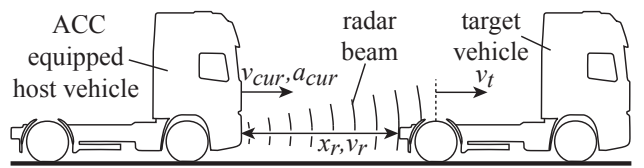

Figure 9: Example of the ACC working principle. The host vehicle, driving with velocity $v_{c u r}$ and acceleration $a_{c u r}$, is equipped with an ACC, which ensures automatic following of the preceding target vehicle, driving with velocity $v_{t}$. A radar measures the relative position $x_{r}$ and the relative velocity $v_{r}=v_{t}-v_{c u r}$ between the vehicles.

\section{Adaptive Cruise Control}

\subsection{Control structure}

Tracking of the optimal velocity trajectory $\hat{v}_{\text {opt }}(x)$ will be difficult for a driver. Therefore, we propose to use an automatic CC system to enable automatic tracking of this trajectory. Moreover, as exact tracking of the optimal velocity trajectory will be impossible in actual traffic, we propose the use of an ACC system [14]. ACC combines CC functionality and automatic following of a preceding vehicle, driving at a lower velocity than the desired CC velocity. In this case, the desired CC velocity is given by the optimal velocity trajectory $\hat{v}_{\text {opt }}(x)$. Possibly preceding traffic is intercepted by the ACC system, switching automatically from $\mathrm{CC}$ functionality to automatic following of this traffic and vice versa. In Fig. 9, a schematic representation of the working principle of an ACC in case of automatic following is shown.

ACC systems typically consist of two parts: a vehicle-independent and a vehicle-dependent control part. The vehicle-independent control part prescribes a desired acceleration trajectory $a_{d}$ for the vehicle. The vehicle-dependent part ensures tracking of this trajectory by determining a correspondingly required power $P_{r e q}$ for the HEV. Assuming that the EMS of the HEV ensures good tracking of $P_{r e q}$, the vehicledependent control part can be regarded as a con- 


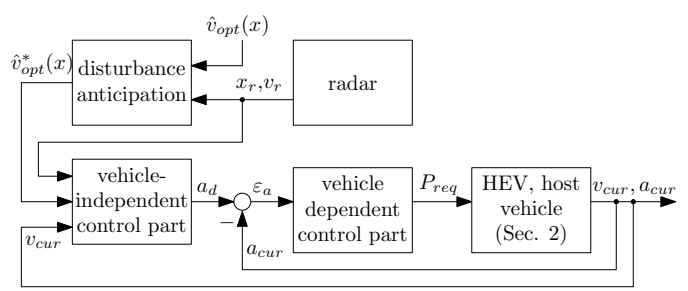

Figure 10: Schematic representation of the ACC control loop. The ACC is divided into a vehicleindependent control loop, determining a desired acceleration $a_{d}$, a vehicle-dependent control loop, determining the required power $P_{r e q}$ for the HEV, and a disturbance anticipation part, adjusting $\hat{v}_{\text {opt }}(x)$ to $\hat{v}_{o p t}^{*}(x)$ for slower driving preceding vehicles. The relative position $x_{r}$ and the relative velocity $v_{r}$ with respect to preceding vehicles are measured using a radar.

troller for the longitudinal vehicle acceleration. In Fig. 10, the ACC control loop is shown. The characteristics of the ACC are determined by the design of the vehicle-independent control part. For this design, the approach presented in [14] is adopted. An explicit MPC approach is employed, to take into account various desirable characteristics, to accommodate constraints, and to provide optimal, situation-dependent controller behavior. As a prediction model, a general vehicle model is adopted.

The desirable characteristics of the ACC are, in this case, fuel economy and safety. The fuel economy is calculated based on a combination of the characteristics of the electric machine, a road-load estimation and the GPS, GIS and route information (see Section 3). Instead of changing the proposed ACC design, an additional control part is designed, enabling anticipation of preceding traffic, i.e. disturbances, that are detected in ample time, in a fuel-economic way. The design of the vehicle-independent control part of the ACC now focuses on safety only. The disturbance anticipation part determines appropriate target velocities, corresponding to the velocity that is prescribed by preceding traffic. Following the approach of Section 3, a corresponding optimal velocity trajectory $\hat{v}_{o p t}^{*}(x)$ is calculated, which replaces the original optimal velocity trajectory $\hat{v}_{\text {opt }}(x)$, and is used as the desired CC velocity. A schematic representation of the resulting setup is shown in Fig. 10.

\subsection{ACC design}

The design of the ACC system comprises the design of the vehicle-independent control part, the vehicle-dependent control part and the disturbance anticipation part (see Figure 10). The design of the vehicle-independent control part and the disturbance anticipation part will be discussed next. For the design of the vehicle- dependent control part, a relatively straightforward PID controller is designed using standard loop shaping techniques. This design will not be discussed further at this point.

\subsection{Vehicle-independent control part}

As discussed in Section 4.1, the vehicleindependent control part is designed following [14], adopting an explicit MPC approach, and focusing on safety. Correspondingly, the control objectives are, firstly, to preserve a desired distance $x_{r, d}(t)$ with respect to a preceding vehicle

$$
x_{r, d}(t)=x_{r, 0}+v_{c u r}(t) h_{d}
$$

where $x_{r, 0}$ the desired distance at standstill and $h_{d}$ the so-called desired time headway, which is the time it takes for the HEV to reach the current position of the preceding target vehicle if the HEV continues to drive with its current velocity [14]. Secondly, the relative velocity between the vehicles, $v_{r}(t)=v_{t}(t)-v_{\text {cur }}(t)$, should be minimized. Besides these objectives, to avoid collisions, a constraint regarding the relative position is defined as $x_{r}(t)>0$.

\subsection{Disturbance anticipation}

The disturbance anticipation part of the ACC system adjusts the optimal velocity trajectory $\hat{v}_{\text {opt }}(x)$, anticipating preceding traffic. Define the current time $t_{0}$ and consider a preceding vehicle driving at a velocity $v_{t}\left(t_{0}\right)$, where $v_{t}\left(t_{0}\right)<$ $\hat{v}_{\text {opt }}\left(x_{0}\right)$, with $x_{0}=x\left(t_{0}\right)$ the position of the HEV at time $t_{0}$. To prevent a future collision, the optimal velocity trajectory has to be adjusted with respect to the velocity of the preceding vehicle. Given the relative velocity $v_{r}\left(t_{0}\right)=v_{t}\left(t_{0}\right)-$ $v_{\text {cur }}\left(t_{0}\right)$, a corresponding time $\Delta t_{b r}$, see (18) and (19), and a displacement $\Delta s_{b r}$, see (22) and (23), that an optimal deceleration would take, can be calculated.

Assume that the relative position between the two vehicles, $x_{r}\left(t_{0}\right)$, is sufficiently large, i.e. the HEV does not need to start decelerating directly to prevent a collision. Define the time at which the deceleration has to start $t_{1}$, the time at which the deceleration ends $t_{2}$, and assume the velocity of the preceding vehicle to be constant, i.e. $v_{t}\left(t_{0}\right)=v_{t}\left(t_{1}\right)=v_{t}\left(t_{2}\right)=v_{t}$. Using (30), the desired distance between the two vehicles at time $t_{2}$ is given by;

$$
\begin{aligned}
x_{r, d}\left(t_{2}\right) & =x_{r, 0}+v_{c u r}\left(t_{2}\right) h_{d} \\
& =x_{r, 0}+v_{t} h_{d}
\end{aligned}
$$

Assuming constant velocity of the HEV and the preceding vehicle, the actual distance at time $t_{2}$ is given by;

$$
\begin{aligned}
x_{r}\left(t_{2}\right) & =x_{r}\left(t_{0}\right)+\left(t_{1}-t_{0}\right)\left(v_{t}-v_{c u r}\left(t_{0}\right)\right) \\
& +v_{t} \Delta t_{b r}-\Delta s_{b r}
\end{aligned}
$$


where $\left.\Delta t\right|_{t_{2}} ^{t_{3}}$ and $\left.\Delta s\right|_{s_{2}} ^{s_{3}}$ as defined in (18), (19) and (22), (23). Equating (31) and (32) yields the time $t_{1}$ till the start of the deceleration;

$$
\begin{gathered}
t_{1}=t_{0} \\
\left(x_{r, d}\left(t_{2}\right)-x_{r}\left(t_{0}\right)+\Delta s_{b r}-v_{t} \Delta t_{b r}\right) v_{r}\left(t_{0}\right)^{-1}
\end{gathered}
$$

The corresponding distance the HEV has to drive till the start of the deceleration is then given by;

$$
x_{h}\left(t_{1}\right)=v_{\text {cur }}\left(t_{0}\right)\left(t_{1}-t_{0}\right)
$$

Hence, the target optimal velocity trajectory with respect to the preceding vehicle is known and the optimal velocity trajectory $\hat{v}_{\text {opt }}(x)$ can be adjusted accordingly, yielding $\hat{v}_{\text {opt }}^{*}(x)$. The ACC system switches from $\mathrm{CC}$ functionality to automatic following when necessary from a point of view of safety, e.g. in case of a sudden cut-in and at small inter-vehicle distances. Note that (18), (19), (22), (23), and (31)-(34), are algebraic relations, and require virtually no computational effort. On a modern notebook computation time is $<0.3 \mathrm{~ms}$.

\section{Predictive Cruise Control setup}

The combination of $i)$ the calculation of the optimal velocity trajectory $\hat{v}_{o p t}(x)$, based on a roadload estimation, the characteristics of the electric machine, and GPS, GIS and routeplanner information, and ii) the ACC system providing automatic tracking of the optimal velocity trajectory as well as attenuation of preceding traffic, is indicated as Predictive Cruise Control (PCC).

In Fig. 11, a schematic representation of the setup of PCC is depicted. The scheme consists of a Route and traffic information part, a Trajectory builder part (as discussed in Section 3 ), an Adaptive Cruise Control part (as discussed in Section 4), and a Vehicle part. The vehicle model was already discussed (see Section 2). The road load estimation is not discussed in this paper, interested readers are referred to, e.g., [11, 18].

The ACC system and the calculation of the optimal velocity trajectory $\hat{v}_{\text {opt }}(x)$ require different sampling times. The ACC system has to react to immediate disturbances, i.e. the actual traffic situation. This requires a sampling frequency in the order of $50 \mathrm{~Hz}$. A fuel optimal deceleration is calculated within this sampling frequency. The optimal velocity trajectory $\hat{v}_{\text {opt }}(x)$ on the other hand, is based on upcoming route information (of the whole route). The trajectory can thus be calculated event driven, that is; once the driver selects a new route. Updating of the total trajectory is time expensive, depending on the number of segments, calculation takes typically several seconds. Therefore, the setup indicates a strict division between the ACC system and the calculation of the optimal velocity trajectory.

\section{Simulation Results}

The setup of PCC as described in the previous section is translated into a Simulink ${ }^{\circledR}$ simulation environment, using the vehicle model of Section 2 and parameters as shown in Table 1 .

The objective of the simulations is to indicate i) the benefit of using an automated $\mathrm{CC}$, ii) the influence of the actual traffic situation on tracking the optimal velocity trajectory. Two ACC actions are simulated:

- Approaching a vehicle driving with a velocity lower than the requested velocity, allowing an optimal deceleration towards the preceding vehicle, see Fig. 12. At the first $3.5 \mathrm{~s}$ of the simulation, the vehicle follows the optimal velocity trajectory. Hereafter the radar output a threshold, and the ACC ensures automatic deceleration. Since the vehicle velocity decreases, the optimal distance $x_{r, d}$ becomes smaller as well. At 15 $\mathrm{s}$ the host vehicle reaches de required distance behind the preceding vehicle. During the deceleration, $0.237 \mathrm{MJ}$ of kinetic energy is recovered.

- A cut-in situation, where the optimal trajectory cannot be followed and the ACC switches to a hard deceleration, see Fig. 13. During the first $9 \mathrm{~s}$ of the simulation, the host vehicle exactly follows the optimal velocity trajectory $\hat{v}_{\text {opt }}(x)$ using the $\mathrm{CC}$ mode. Then suddenly a preceding vehicle cuts in in front of the host vehicle, as can be seen in the middle figure. Optimal deceleration is impossible, therefore the host vehicle applies hard braking, to avoid a collision.

\section{Conclusions}

Earlier work [10] indicated that route optimization by using map data can provide a considerable fuel consumption improvement in heavyduty HEV. However, route optimization has only practical relevance when the driver can be assisted in following the optimal trajectory, and the optimization can adapt to the current traffic situation.

In this paper analytical solutions are derived for the velocity trajectory optimization problem, that exploits the HEV specific opportunity of kinetic energy recovery. Calculation of optimal deceleration trajectory proves to be fast enough for ACC implementation. The ACC is constructed such that the combination of optimal velocity trajectory construction and ACC is presented as PCC. Simulations show that PCC is a suitable aid to implement optimal driving courses in practice.

\section{Outlook on future research}

There are several parts of the PCC setup that require further research. Firstly, the trajectory 


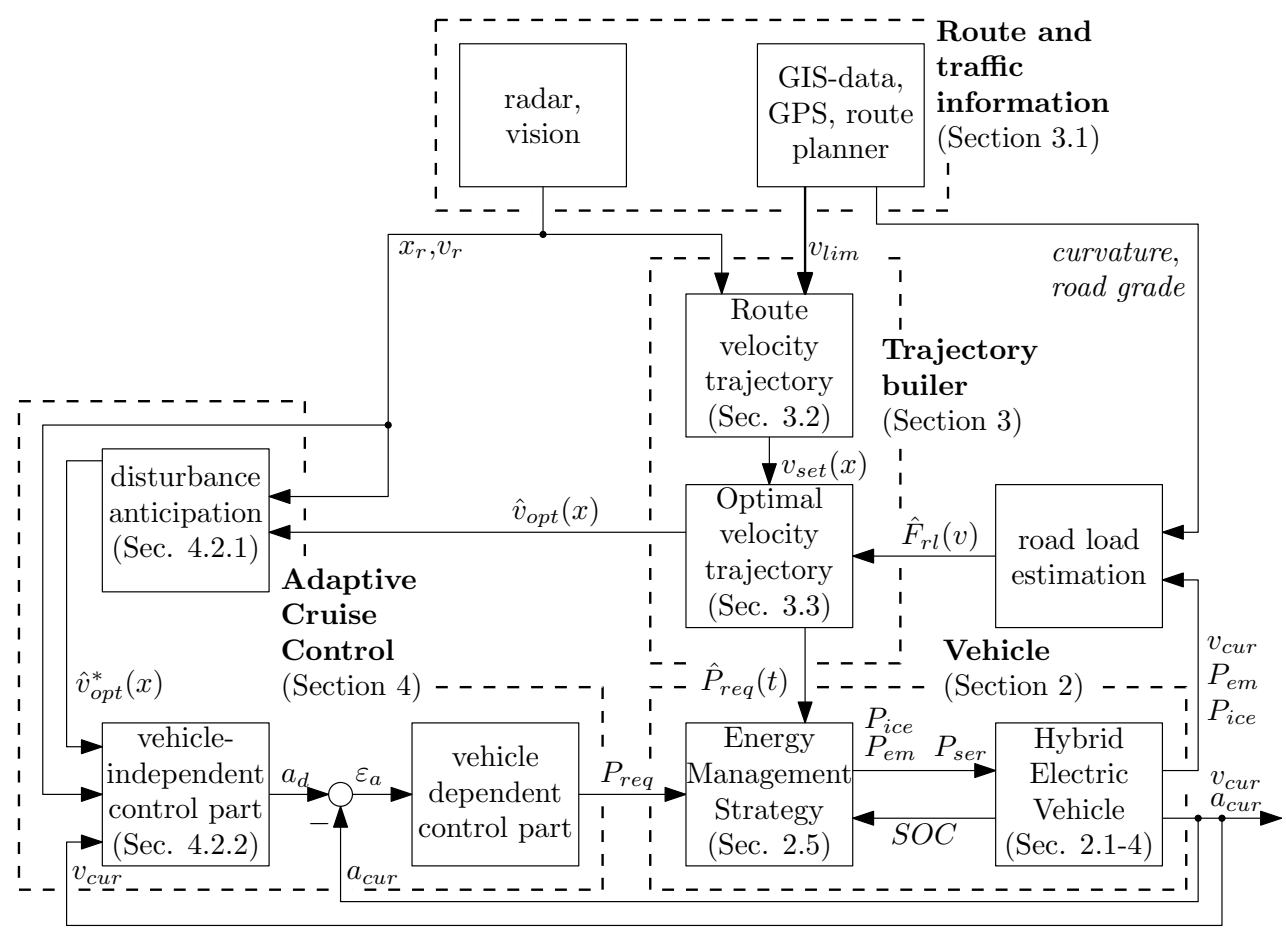

Figure 11: Schematic representation of the setup of Predictive Cruise Control. Legend: $x_{r}$ is the relative distance to the preceding vehicle (or stopping point), $v_{r}$ is the relative velocity between the preceding vehicle and the host vehicle, $v_{\text {lim }}$ is the velocity limitation, $v_{\text {set }}(x)$ is the route matrix containing relevant segment parameters, $\hat{F}_{r l}(v)$ is the road load force estimation, $\hat{v}_{\text {opt }}(x)$ is the optimized velocity trajectory as function of distance, $\hat{v}_{o p t}^{*}$ is the adjusted optimal velocity trajectory, $v_{c u r}$ is the current vehicle velocity, $a_{c u r}$ is the current vehicle acceleration, $a_{d}$ is the acceleration setpoint, $\varepsilon_{a}$ is the acceleration error, $P_{r e q}$ is the power request, $\hat{P}_{r e q}(t)$ is the predicted future power request trajectory, $P_{s e r}$ is the brake power of the service brakes, $P_{e m}$ is the power of the electric machine, $P_{i c e}$ is the power of the internal combustion engine, $S O C$ is the current battery state-of-charge, $a_{c u r}$ is the current vehicle acceleration, and finally $v_{c u r}$ is the current vehicle velocity.

builder can be extended with route optimization subject to a time constraint, including also road grading. Secondly, verification of the simulation results with a test vehicle on the university chassis dynamo meter is scheduled. Thirdly, when the automated CC is shut off, and the driver is driving, the signal $a_{d}$ can be used for driver assistance. For instance by force feedback on the brake pedal, or an indication on the dashboard. Furthermore, PCC system could recognize parts in the route where automated $\mathrm{CC}$ is impossible. For example due to;

- approaching a traffic light without traffic light status information,

- approaching a intersection without right of way,

- unclear map data,

- GPS failure.

Finally, application of vision to recognize stopping points (traffic light status) will be investigated.

\section{Acknowledgement}

The research presented in this paper is part of a more extensive project in the development of advanced energy management control for urban distribution trucks which has been made possible by TNO Business Unit Automotive in cooperation with DAF Trucks NV.

\section{References}

[1] D. A. Abbink, E. R. Boer, M. Mulder Motivation for Continuous Haptic Gas Pedal Feedback to Support Car Following, IEEE Intelligent Vehicles Symposium, Eindhoven, The Netherlands, June 4-6, 2008.

[2] H. Bruneel. Heavy Duty Testing Cycles Development: a New Methology, International Spring Fuels \& Lubricants Meeting \& Exposi- 


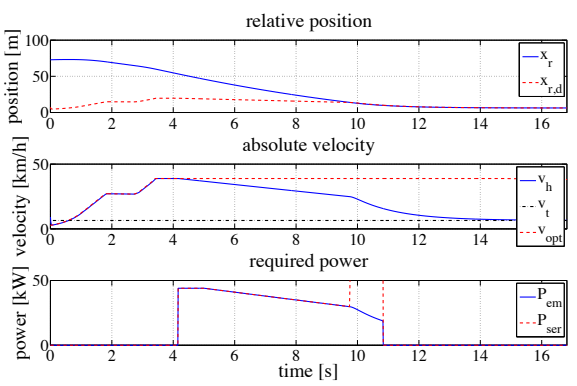

Figure 12: Adaptive Cruise Control simulation results of vehicle approach. The upper figure shows the relative distance between the host vehicle and a preceding vehicle (solid), and the requested distance to a preceding vehicle (dashed). The middle figure shows the velocity of the host vehicle (solid), velocity of the preceding vehicle (dash-dotted), the precalculated optimal velocity trajectory (dashed). The lower figure shows the power usage of the electric machine and/or service brakes.

tion, (SAE 2000-01-1890), Paris, France, June 19-20, 2000.

[3] L. Guzzella, A. Sciarretta. Vehicle Propulsion Systems, ISBN 3-540-25195-2, SpringerVerlag, Berlin, Heidelberg, 2005.

[4] E. Hellstöm, M. Ivarsson, J. Åslund, L. Nielsen. Look-ahead control for heavy trucks to minimize trip time and fuel consumption, Control Engineering Practice 17, pp. 245-254, July 2008.

[5] I. Husain. Electric and Hybrid Vehicles: Design Fundamentals, ISBN 0-8493-1466-6, CRC Press, 2003.

[6] L. Johannesson, M. Åsbogård, B. Egardt. Assessing the Potential of Predictive Control for Hybrid Vehicle Powertrains Using Stochastic Dynamic Programming IEEE Transactions on Intelligent Transportation Systems, Vol. 8, No. 1, pp 71-83, March 2007.

[7] K. Li, H.-S. Tan, J.K. Hedrick. An Enhanced GPS-Based Vehicle Positioning System through Sensor Fusion of Digital Map Data, AVEC'08 International Symposium on Advanced Vehicle Control, Kobe, Japan, pp. 479-484, October 2008.

[8] J.T.B.A. Kessels, P.P.J. van den Bosch. Electronic horizon: road information used by Energy Management Strategies International Journal Intelligent Information and Database Systems, Vol. 2, No.2, pp. 187-203, 2008.

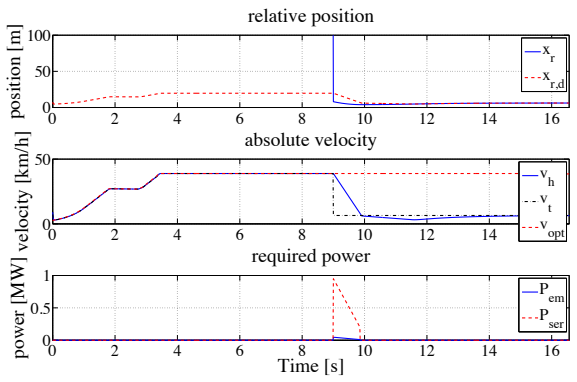

Figure 13: Adaptive Cruise Control simulation results of vehicle cut in. The upper figure shows the relative distance between the host vehicle and a preceding vehicle (solid), and the requested distance to a preceding vehicle (dashed). The middle figure shows the velocity of the host vehicle (solid), velocity of the preceding vehicle (dash-dotted), the precalculated optimal velocity trajectory (dashed). The lower figure shows the power usage of the electric machine and/or service brakes.

[9] T. van Keulen, B. de Jager, M. Steinbuch. Influence of Driver, Route and Vehicle Mass on Hybrid Electric Truck Fuel Economy, AVEC'08 International Symposium on Advanced Vehicle Control, Kobe, Japan, pp. 911-916, October 2008.

[10] T. van Keulen, B. de Jager, A. Serrarens, M. Steinbuch. Energy Management in Hybrid Electric Trucks Using Route Information, to be printed in Oil \& Gas Science and Technology.

[11] I. Kolmanovsky, V. Winstead. A Receding Horizon Optimal Control Approach to Active State and Parameter Estimation in Automotive Systems. Proceedings of the 2006 IEEE International Conference on Control Applications, pp. 2796-2801, Munich, Germany, October 4-6, 2006.

[12] J.Ph. Lauffenburger, B. Bradai, M. Basset, F. Nashashibi. Navigation and Speed Signs Recognition Fusion for Enhanced Vehicle Location, Proc. of the 17th IFAC World Congress, pp 2069-2074, Seoul, Korea, July 6-11 2008.

[13] V. Monastyrsky, I. Golownykh. Rapid computation of optimal control for vehicles, Transportation Research, Vol. 27B, No. 3, pp. 219-227, 1993.

[14] G.J.L. Naus, R.A.P.M. van den Bleek, J. Ploeg, B. Scheepers, M.J.G. van de Molengraft, M. Steinbuch. Explicit MPC design and performance evaluation of an ACC Stop-\&-Go, 2008 
American Control Conference, pp. 224-229, WA, United States, June 2008.

[15] P. Pisu, G. Rizzoni. A Comparative Study Of Supervisory Control Strategies for Hybrid Electric Vehicles, IEEE Transactions on Control Systems Technology, Vol. 15, No. 3, pp. 506-518, May 2007.

[16] A. Sciarretta, L. Guzzella. Control of Hybrid Electric Vehicles, IEEE Control Systems Magazine, Vol. 27, No. 2, pp. 60-70, April 2007.

[17] A.P. Stoicescu. On Fuel-Optimal Velocity Control of a Motor Vehicle, International Journal of Vehicle Design, Vol. 16, Nos 2/3, pp. 229-256, 1995.

[18] A. Vahidi, A. Stefanopoulou, H. Peng. Recursive least squares with forgetting for online estimation of vehicle mass and road grade: theory and experiments, Vehicle System Dynamics, Vol. 43, No. 1, pp. 31-55, January 2005.
Table 1: Simulation parameters for medium-sized heavy-duty HEV.

\begin{tabular}{|c|c|c|c|}
\hline \multicolumn{4}{|c|}{ Vehicle parameters } \\
\hline Name & Description & Value & Unit \\
\hline$\rho_{a}$ & air density & 1.29 & $\mathrm{~kg} / \mathrm{m}^{3}$ \\
\hline$A_{f}$ & frontal area & 7.68 & $\mathrm{~m}^{2}$ \\
\hline$c_{a}$ & aerodynamic coef. & 0.673 & - \\
\hline$c_{d}$ & differential coef. & 0.225 & $\mathrm{Ns} / \mathrm{m}$ \\
\hline$c_{d 0}$ & diff. initial coef. & 5 & $\mathrm{~N}$ \\
\hline$c_{g b}$ & gearbox coef. & 0.225 & $\mathrm{Ns} / \mathrm{m}$ \\
\hline$c_{g b 0}$ & gearbox ini. coef. & 10 & $\mathrm{~N}$ \\
\hline$c_{r}$ & rolling res. coef. & 0.0075 & - \\
\hline$g$ & gravitat. const. & 9.81 & $\mathrm{~m} / \mathrm{s}^{2}$ \\
\hline$k$ & crosswind coef. & 0.17 & - \\
\hline$i_{f}$ & final drive ratio & 5.13 & - \\
\hline$i_{g b}$ & gearbox ratio & - & - \\
\hline$m$ & vehicle mass & 8320 & $\mathrm{~kg}$ \\
\hline$m_{e}$ & effective mass & 8695 & $\mathrm{~kg}$ \\
\hline$r_{e}$ & tire eff. radius & 0.52 & $\mathrm{~m}$ \\
\hline \multicolumn{4}{|c|}{ Trajectory builder parameters } \\
\hline$k_{i c e}$ & aver. combustion eff. & 2.35 & $\mathrm{~kJ} / \mathrm{s}$ \\
\hline$h_{f}$ & lower heating value & 42.7 & $\mathrm{~kJ} / \mathrm{g}$ \\
\hline$\dot{m}_{f 0}$ & idle fuel cons. & 0.4 & $\mathrm{~g} / \mathrm{s}$ \\
\hline$P_{\max }$ & max. acc. power & 125 & $\mathrm{~kW}$ \\
\hline$T_{d r a g}$ & engine drag torque & 150 & $\mathrm{Nm}$ \\
\hline$T_{e m}$ & EM max. torque & 420 & $\mathrm{Nm}$ \\
\hline$P_{e m}$ & EM max. power & 44 & $\mathrm{~kW}$ \\
\hline \multicolumn{4}{|c|}{ ACC parameters } \\
\hline$h_{d}$ & time headway & 1.5 & $\mathrm{~s}$ \\
\hline$x_{r, d}$ & desired dist. & - & $\mathrm{m}$ \\
\hline$x_{r, 0}$ & des. dist. at $v_{c u r}=0$ & 3.5 & $\mathrm{~m}$ \\
\hline \multicolumn{4}{|c|}{ Route parameters } \\
\hline$\beta$ & road grade & - & $\mathrm{rad}$ \\
\hline$R$ & road curvature & - & $\mathrm{m}$ \\
\hline$v$ & vehicle speed & - & $\mathrm{m} / \mathrm{s}$ \\
\hline$v_{x}$ & wind velocity & 0 & $\mathrm{~m} / \mathrm{s}$ \\
\hline$v_{y}$ & side wind vel. & 0 & $\mathrm{~m} / \mathrm{s}$ \\
\hline
\end{tabular}

